\title{
Puesta al día en el estudio de susceptibilidad de Streptococcus pneumoniae
}

\author{
ELIZABETH PALAVECINO R.* \\ UPDATE ON in vitro SUSCEPTIBILITY TESTING OF \\ Streptococcus pneumoniae
}

Key words: Streptococcus pneumoniae, Susceptibility testing, Break points.

\section{INTRODUCCIÓN}

Aunque la resistencia a penicilina en Streptococcus pneumoniae fue descrita en 1971, este microorganismo permaneció susceptible hasta hace un poco más de 10 años atrás cuando se comenzó a observar un tremendo incremento en su resistencia a penicilina. Este fenómeno ha sido descrito prácticamente en todo el mun- do y ha obligado a los laboratorios clínicos a realizar estudio de susceptibilidad en forma rutinaria en $S$. pneumoniae. (Tabla 1).

Los puntos de corte que inicialmente propuso el Comité Nacional de Estandarización de los Laboratorios Clinicos (National Committee for Clinical Laboratory Standards-NCCLS) para la interpretación de la susceptibilidad de $S$. pneumoniae fueron basados en los casos de

Tabla1. Puntos críticos en el estudio de susceptibilidad en Streptococcus pneumoniae

- Las cepas obtenidas de sitios estériles y muestras respiratorias deben ser estudiadas de rutina.

- Los antimicrobianos a estudiar varían según el sitio de aislamiento

- En cepas obtenidas de LCR use un método de CIM e informe penicilina, ceftriaxona o cefotaxima, vancomicina y meropenem

- En cepas de infecciones respiratorias haga un screening usando un disco de oxacilina para estudiar susceptibilidad a penicilina, confirme por epsilometría (E-test $\left.{ }^{\circledR}\right)$ si el área de inhibición es $<19 \mathrm{~mm}$.

- Los macrólidos y las quinolonas pueden también ser estudiados mediante difusión por disco.

- Las cefalosporinas NO deben ser estudiadas mediante difusión por disco.

- Amoxicilina puede ser estudiada por un método de CIM e informada en infecciones respiratorias.

\footnotetext{
* Department of Pathology, University Hospitals of Cleveland. Case Western Reserve University Cleveland OH, USA.
} 
meningitis causados por cepas resistentes a penicilina, (criterios muy conservadores), con el fundamento que la mayoría de los agentes antimicrobianos no alcanzan altas concentraciones en el LCR.

Sin embargo, en los últimos años numerosos estudios han tratado de establecer una mejor correlación entre los puntos de corte y respuesta clínica en infecciones que no son meníngeas, como es el caso de otitis media aguda, exacerbaciones de bronquitis crónica y neumonía. Es indudable que la respuesta clínica no sólo depende de la concentración mínima del antimicrobiano a la cual el microorganismo es inhibido (CIM) sino que también depende de la farmacodinamia y farmacocinética del agente antibacteriano, del estado immunológico del paciente y del sitio en que se ubica la infección. En el caso de $S$. pneumoniae, este concepto ha traído mucha controversia ya que la actividad de los diferentes antimicrobianos usados para tratar infecciones producidas por neumococos resistentes varía sustancialmente dependiendo del lugar de la infección. ${ }^{1,2}$

En esta revisión entregaremos pautas para realizar el estudio de susceptibilidad en S. pneumoniae de acuerdo a las estandarizaciones actuales, discutiremos la interpretación de los resultados del estudio de susceptibilidad y daremos a conocer los nuevos puntos de corte. En la Tabla 2 se detallan las especificaciones de los métodos y medios a usar con cada uno de ellos.

Conceptos de PK/PD (pharmacokinetics/ pharmacodinamics-farmacinética/farmaco- dinamia). La eficacia de todos los $\beta$ lactámicos (penicilinas y cefalosporinas) y de los macrólidos (excepto azitromicina, estrictamente una azálida) depende del tiempo durante el cual el fármaco se mantiene en el sitio de la infección a una concentración que sobrepasa la CIM del microorganismo causante. Una buena respuesta clínica se ha observado cuando la concentración local de los $\beta$ lactámicos supera la CIM al menos durante 40 a $50 \%$ del intervalo de tiempo entre dos dosis sucesivas. Si se toman en cuenta las dosis terapéuticas diarias se puede anticipar la concentración que un determinado antibacteriano mantendrá por sobre la CIM de un microorganismo en particular durante $40 \%$ del lapso de tiempo entre una dosis y la siguiente. Esta concentración es el punto de corte del PK/PD. ${ }^{1}$

Los parámetros de $\mathrm{PK} / \mathrm{PD}$ en el caso de quinolonas y de azitromicina son diferentes a los ya mencionados, en este caso la eficacia depende más bien de la concentración máxima del agente y su relación con la CIM del microorganismo.

La discusión completa de los puntos de corte obtenidos por los parámetros de PK/PD escapa al objetivo de este artículo, sólo quisiéramos destacar que los puntos de corte del NCCLS pueden ser en algunos casos muy diferentes de los puntos de corte derivados de los estudios basados en los parámetros de PK/PD. ${ }^{1,2}$ Por este motivo el NCCLS ha tenido que cambiar o eliminar los puntos de corte de algunos agentes (como sucedió con las cefalosporinas orales). Más recientemente muchos médicos clínicos y

Tabla 2. Métodos recomendados para el estudio de susceptibilidad in vitro en Streptococcus pneumoniae

\begin{tabular}{|c|c|c|c|}
\hline Método & Medio & Inóculo & Incubación \\
\hline Microdilución en caldo & $\begin{array}{l}\text { Caldo de Mueller-Hinton con 2-5\% } \\
\text { sangre hemolizada de caballo }\end{array}$ & $\begin{array}{l}\text { Suspensión directa } \\
0,5 \mathrm{McFarland}\end{array}$ & $\begin{array}{l}35^{\circ} \mathrm{C} \text {, en aire } \\
\text { durante } 20-24 \text { horas }\end{array}$ \\
\hline Difusión por discos & $\begin{array}{l}\text { Agar de Mueller-Hinton con 5\% } \\
\text { sangre de cordero }\end{array}$ & $\begin{array}{l}\text { Suspensión directa } \\
0,5 \mathrm{McFarland}\end{array}$ & $\begin{array}{l}35^{\circ} \mathrm{C} \text { en } 5 \% \mathrm{CO}_{2} \\
\text { durante } 20-24 \text { horas }\end{array}$ \\
\hline E-test ${ }^{\circledR}$ & $\begin{array}{l}\text { Agar de Mueller-Hinton con } 5 \% \\
\text { sangre de cordero }\end{array}$ & $\begin{array}{l}\text { Suspensión directa } \\
0,5 \text { McFarland }\end{array}$ & $\begin{array}{l}35^{\circ} \mathrm{C} \text { en } 5 \% \mathrm{CO}_{2} \\
\text { durante } 20-24 \text { horas }\end{array}$ \\
\hline
\end{tabular}


microbiólogos han estado presionando para que el NCCLS agregue una interpretación basada en el sitio de la infección. Aunque en teoría esto es lo que se debe hacer ya que tiene mayor correlación con la respuesta clínica, en la práctica es difícil de llevar a cabo porque los laboratorios clínicos tendrían que, no sólo detectar las cepas resistentes sino que también, informar la susceptibilidad tomando en cuenta los diferentes puntos de corte según el sitio de aislamiento; todo ello significa aumentar enormemente el trabajo rutinario al cual es sometido el personal. El nuevo documento del NCCLS, (M100-S12 del año 2002) incluye puntos de corte para algunos agentes de acuerdo al sitio de infección: meningitis y no-meningitis (Tabla 3 ).

Susceptibilidad a penicilinas. La resistencia a penicilina y a los otros $\beta$ lactámicos en $S$. pneumoniae es debida a modificaciones en las PBPs, lo que produce una menor afinidad entre estas proteínas y el agente antimicrobiano.

Un enfoque muy práctico es usar un disco de oxacilina de $1 \mu \mathrm{g}$ para deducir la susceptibilidad a penicilina. Si la zona de inhibición en rededor al disco de oxacilina es $>20 \mathrm{~mm}$, la cepa es considerada susceptible a penicilina y se puede informar como tal. Si el área de inhibición es $<19 \mathrm{~mm}$ esa cepa debe ser estudiada por un método cuantitativo de CIM para clarificar si es susceptible, tiene susceptibilidad intermedia o es resistente a penicilina, ya que un pequeño porcentaje de cepas tendrá un área $<19$ mm pero puede todavía ser susceptible.

Los puntos de corte para definir susceptibilidad y resistencia de $S$. pneumoniae a penicilina por CIM siguen siendo los mismos: una cepa con CIM de $<0,06 \mu \mathrm{g} / \mathrm{ml}$ es susceptible, con CIM de 0,12 a 1,0 es intermedia y con una CIM $\mathrm{de}>2 \mu \mathrm{g} / \mathrm{ml}$ es considerada resistente a penicilina. Si un neumococo es susceptible a penicilina, debe ser considerado susceptible también a otras penicilinas.

Con respecto a amoxicilina, el NCCLS cambió en enero del 2000 los puntos de corte, basado en nueva información relativa a farmacodinamia y farmacocinética de este agente. La amoxicilina sólo puede ser estudiada por un método de CIM y no debe ser evaluada si la cepa ha sido aislada de LCR en un paciente con meningitis, ya que estos puntos de corte son para infecciones respiratorias. Los nuevos pun- tos de corte son: CIM de $<2 \mu \mathrm{g} / \mathrm{ml}$ indica susceptibilidad, CIM de $4 \mu \mathrm{g} / \mathrm{ml}$ indica susceptibilidad intermedia y CIM de > a $8 \mu \mathrm{g} / \mathrm{ml}$ es considerada resistente. En consecuencia, cepas de neumococos que presentan susceptibilidad intermedia o resistencia a penicilina pueden ser todavía susceptibles a amoxicilina. Además, si un neumococo es aislado de un paciente con neumonía y presenta susceptibilidad intermedia a penicilina, puede ser tratado aún con penicilina, usando dosis altas de este agente. ${ }^{3}$

Susceptibilidad a cefalosporinas. Como comentáramos anteriormente, la resistencia a cefalosporinas es producida también por modificaciones de las PBPs que se encuentran en la pared celular del neumococo. Las cefalosporinas de tercera generación son muy eficaces en el tratamiento de infecciones producidas por $S$. pneumoniae, estos agentes deben ser estudiados e informados en todas las cepas aisladas de LCR. Este año el NCCLS ha incluido puntos de corte diferentes dependiendo de si la cepa ha sido aislada de un paciente con meningitis o no. Las cefalosporinas que tienen estos nuevos puntos de corte son: ceftriaxona, cefotaxima y cefepime. Sin embargo, es necesario tener en cuenta que cefepime no ha sido autorizado aún por la FDA para el tratamiento de meningitis causadas por $S$. pneumoniae resistente; por esto en la actualidad, el NCCLS recomienda informarlo sólo en aquellas infecciones no meníngeas. (Tabla 3).

En cambio, las cefalosporinas orales varían bastante en su eficacia contra $S$. pneumoniae como se puede ver en la Figura 1. Algunas de las cefalosporinas orales no alcanzan concentraciones suficientemente altas en el sitio de infección para inhibir cepas causantes de infecciones respiratorias con susceptibilidad intermedia o resistentes a penicilina. No existe hasta este momento una estandarización ni tampoco puntos de corte para estudiar las cefalosporinas mediante el método de difusión por disco y por lo tanto, estos agentes no deben ser estudiado por este método. Además, como hemos discutido en el caso de Haemophilus influenzae, es muy importante considerar la farmacodinamia y la farmacocinética de las diferentes cefalosporinas orales, así como también el sitio de la infección, antes de decidir la terapia más adecuada. 
Puesta al día en el estudio de susceptibilidad de Streptococcus pneumoniae - E. Palavecino R.

Tabla 3. Puntos de corte para la interpretación del estudio de susceptibilidad in vitro en Streptococcus pneumoniae a seleccionados grupos de antimicrobianos (NCCLS 2002)

\begin{tabular}{lllll}
\hline $\begin{array}{l}\text { Agente } \\
\text { Antimicrobiano }\end{array}$ & $\mathbf{S}$ & $\begin{array}{c}\text { CIM }(\boldsymbol{\mu g} / \mathbf{m l}) \\
\mathbf{I}\end{array}$ & $\mathbf{R}$ & \multicolumn{1}{c}{ Comentarios } \\
Penicilina & $<0,06$ & $0,12-1$ & $>2$ & $\begin{array}{l}\text { Una cepa susceptible a penicilina es también } \\
\text { susceptible a otras penicilinas }\end{array}$ \\
Amoxicilina & $<$ & & & No evalúe en meningitis \\
Cefepime & & & & \\
(no-meningitis) & $<1$ & 2 & $>4$ & Informe sólo en infecciones no meníngeas \\
(meningitis) & $<0,5$ & 1 & $>2$ & \\
Cefotaxima/ceftriaxona & & & \\
(no-meningitis) & $<1$ & 2 & $>4$ & En cepas aisladas de LCR, informe sólo \\
(meningitis) & $<0,5$ & 1 & $>2$ & la interpretacion de meningitis \\
Meropenem & $<0,25$ & 0,5 & $>1$ & \\
Imipenem & $<0,12$ & $0,25-0,5$ & $>1$ & \\
Eritromicina & $<0,25$ & 0,5 & $>1$ & Existe resistencia cruzada. Si una cepa es \\
Claritromicina & $<0,25$ & 0,5 & $>1$ & resistente a un macrólido, también es resistente \\
Azitromicina & $<0,5$ & 1 & $>2$ & a los otros miembros de este grupo \\
Clindamicina & $<0,25$ & 0,5 & $>1$ & \\
Ofloxacina & $<2$ & 4 & $>8$ & Las cepas resistentes son aún poco frecuentes. \\
Levofloxacina & $<2$ & 4 & $>8$ & Confirme esta resistencia \\
Gatifloxacina & $<1$ & 2 & - & No han sido descritas cepas resistentes \\
Vancomicina & $<1$ & - & &
\end{tabular}

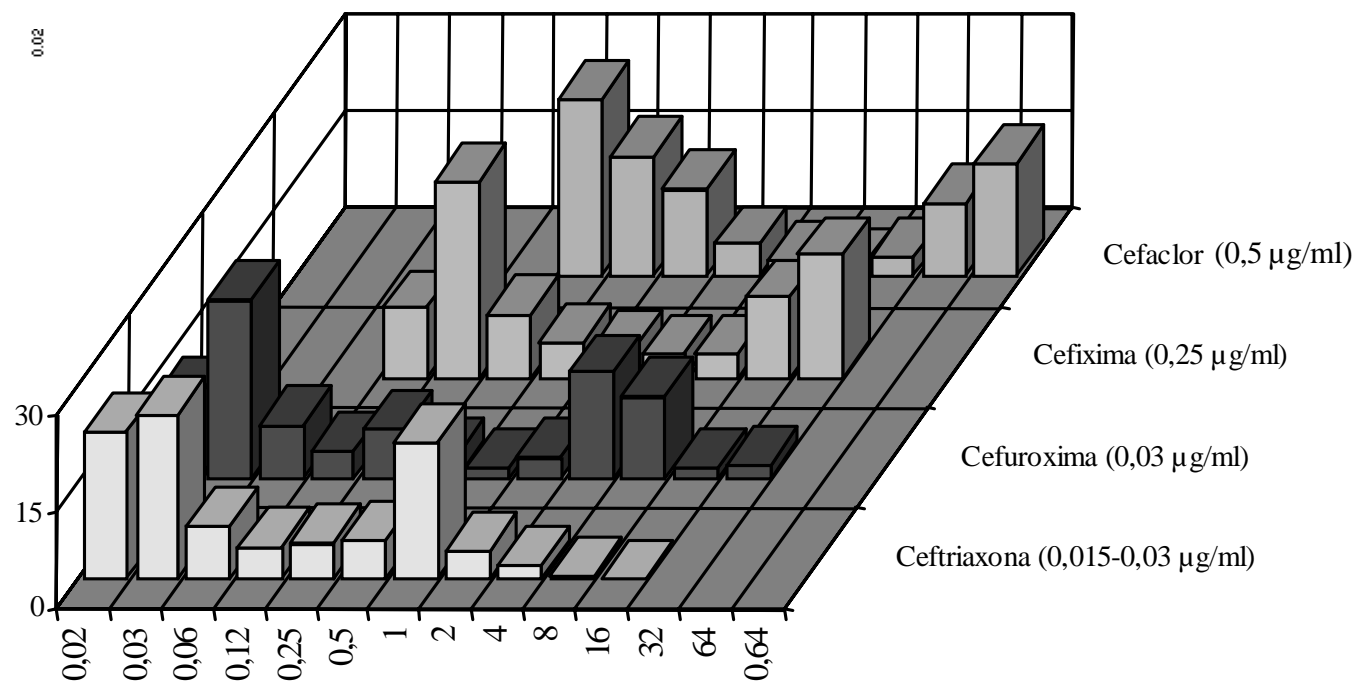

Figura 1. Distribución de CIMs a varias cefalosporinas en Streptococcus pneumoniae. Nótese la diferencia en actividad entre estos agentes. Ceftriaxona y cefuroxima inhiben la mayoría de las cepas de $S$. pneumoniae a concentraciones de 0,03 - 0,06 $\mu \mathrm{g} / \mathrm{ml}$. En cambio cefaclor necesita concentraciones de $0,5 \mu \mathrm{g} / \mathrm{ml}$ para inhibir cepas de S.pneumoniae que son susceptibles. (Adaptado de referencia 4). 
Susceptibilidad a macrólidos. La resistencia a macrólidos ha ido en aumento en muchos países. Nosotros encontramos que entre 1997 y 1999 la prevalencia de cepas de S. pneumoniae resistentes a macrólidos era de aproximadamente $8 \%$ en Santiago, Chile, similar a la resistencia encontrada en Streptococcus pyogenes. ${ }^{4}$ El mejor método para estudiar la susceptibilidad de $S$. pneumoniae a macrólidos es el método de difusión por disco, usando una placa de agar de Mueller-Hinton con 5\% de sangre. ${ }^{5}$ Para diferenciar los distintos fenotipos de resistencia a macrólidos se puede incluir un disco de clindamicina. Este disco debe ponerse a una distancia de $16 \mathrm{~mm}$ (de borde a borde) del disco de eritromicina. Si la cepa es resistente a los dos antibacterianos, ya sea con resistencia inducible o constitutiva, ese microorganismo presenta una resistencia del tipo $\mathrm{MLS}_{\mathrm{B}}$, mediada por el gen erm, y será resistente a todos los macrólidos, lincosamidas y estreptograminas. Si el microorganismo sólo es resistente a eritromicina y no a clindamicina, se tratará de una cepa tipo $\mathrm{M}$ de resistencia a macrólidos, mediada por el gen mef, siendo resistente sólo a macrólidos. Estos tipos de resistencia están descritos con más detalle en el artículo sobre $S$. pyogenes (Artículo relacionado en página $\mathrm{S} 107$ ).

Si una cepa de $S$. pneumoniae es resistente a eritromicina, esa cepa será también resistente a los otros macrólidos: claritromicina y azitromicina. En general, los neumococos que presentan el fenotipo $\mathrm{M}$ de resistencia a macrólidos tienen una CIM entre 8 y $16 \mu \mathrm{g} / \mathrm{ml}$, en cambio los neumococos con el fenotipo $\mathrm{MLS}_{\mathrm{B}}$ presentan CIM por sobre $32 \mu \mathrm{g} / \mathrm{ml}$. (Figura 2).

Susceptibilidad a quinolonas. En general, $S$. pneumoniae es muy susceptible a las nuevas quinolonas. Sin embargo, ya han sido reportadas en la literatura fallas al tratamiento con fluoroquinolonas en infecciones producidas por $S$. pneumoniae. La resistencia a quinolonas es producida por varios mecanismos incluyendo mutaciones en las enzimas ADN girasa y en la topoisomerasa IV, las que participan en la síntesis del ADN bacteriano. A mayor número de mutaciones, mayor es la CIM; por lo tanto, si sólo una mutación está presente, la CIM aumenta sólo un poco y es dificil de detectar. En cambio, si han ocurrido varias mutaciones, tanto en la ADN girasa como en la topoisomerasa, la CIM es bastante alta. Hasta el momento no se ha definido cuál es el mejor antimicrobiano de este grupo que detecte en forma eficiente estas mutaciones y que debiera usarse para hacer el estudio in vitro. Sí sabemos que de los casos de resistencia descritos en la literatura, ésta no fue detectada en todas las ocasiones por el método de difusión en disco. Por el mo-

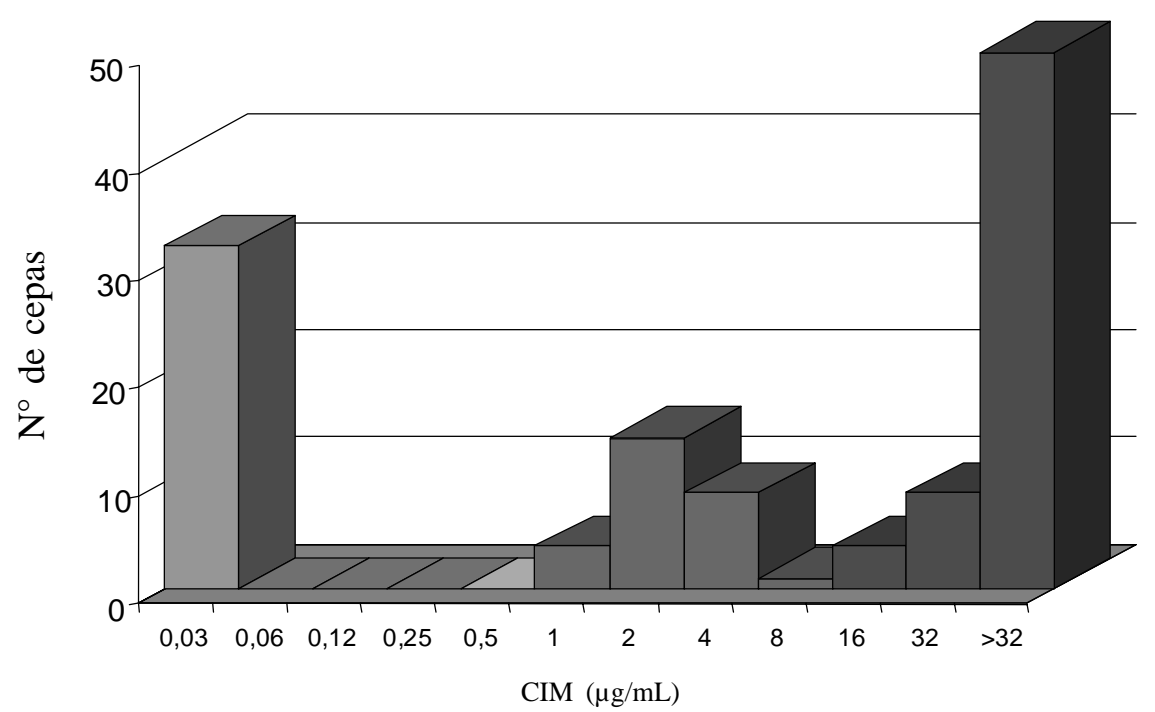

Figura 2. Distribución de CIMs a eritromicina en Streptococcus pneumoniae. Se pueden observar tres diferentes poblaciones: susceptible (con CIM $<0,06 \mu \mathrm{g} / \mathrm{ml}$ ), con fenotipo $\mathrm{M}$ de resistencia (CIM de $>1 \mathrm{a} 16 \mu \mathrm{g} / \mathrm{ml}$ ), y con fenotipo $\mathrm{MLS}_{\mathrm{B}}$ de resistencia a macrólidos (CIM de $>32 \mu \mathrm{g} / \mathrm{ml}$ ). 
mento, el NCCLS recomienda usar un disco de ofloxacina para predecir la susceptibilidad de $S$. pneumoniae a las diferentes quinolonas. Si una cepa es susceptible a ofloxacina, es también susceptible a las nuevas fluoroquinolonas (levofloxacina, gatifloxacina, moxifloxacina y gemifloxacina) En mi opinión, y debido al bajo número de cepas resistentes detectadas hasta ahora, no es necesario estudiar las quinolonas en forma rutinaria.

Susceptibilidad a otros antimicrobianos. Ultimamente ha habido un creciente interés en desarrollar nuevos agentes para tratar infecciones producidas por $S$. pneumoniae resistente. Por el momento no es necesario realizar estudio de susceptibilidad en forma rutinaria, ya que no existen puntos de corte para la interpretación de los resultados. La susceptibilidad puede ser inferida de estudios publicados en la literatura. ${ }^{6}$

\section{RESUMEN DE RECOMENDACIONES}

- El estudio de susceptibilidad en S. pneumoniae debe hacerse en forma rutinaria usando las más recientes recomendaciones y puntos de corte.

- Como se ha discutido anteriormente, se debe hacer primero un screening con un disco de oxacilina para obtener la susceptibilidad a penicilina (excepto en aquellos $S$. pneumoniae aislados de LCR) usando el medio especificado en la Tabla 1. En esta misma placa se pueden agregar discos de eritromicina y clindamicina. Recuerde que las cefalosporinas no deben ser estudiadas por difusión en agar.

- Si el área de inhibición en rededor del disco de oxacilina es $<19 \mathrm{~mm}$, se debe confirmar la resistencia por el método de epsilometría (E-test $\left.{ }^{\circledR}\right)$ usando una tira de penicilina. Se puede agregar además una tira de ceftriaxona o cefotaxima, en especial si se han detectado previamente cepas de neumococos resistentes a cefalosporinas en ese hospital.

- Si la cepa de S. pneumoniae ha sido aislada de LCR, es aconsejable recurrir de inmediato a la epsilometría (u otro método de CIM) para estudiar la susceptibilidad a penicilina y a ceftriaxona (o cefotaxima). En la misma placa se puede agregar un disco de vancomicina. Además el NCCLS recomienda hacer el estudio de susceptibilidad a meropenem por un método de CIM. Si la prevalencia de cepas resistentes a cefalosporinas en un determinado hospital es muy baja, en mi opinión no es absolutamente necesario hacer el estudio a meropenem.

- Para el control de calidad de los diferentes métodos debe usarse la cepa ATCC de Streptococus pneumoniae 49619. La Tabla 3 describe los puntos de corte para algunos antimicrobianos. Refiérase a las tablas del NCCLS para obtener los rangos aceptables para antimicrobianos adicionales. ${ }^{3}$

\section{BIBLIOGRAFÍA}

1.- PALAVECINO E L. The crisis of the resistant pathogens in respiratory tract infections-Use of pharmacodynamic principles. Am J Managed Care 2001; 7: S170-S177.

2.- JACOBS M R, BAJAKSOUZIAN S, ZILLES A, LIN G, PANKUCH G, APPELBAUM P C. Susceptibilities de Streptococcus pneumoniae and Haemophilus influenzae to 10 oral antimicrobial agents based on pharmacodynamic parameters: 1997 U.S. surveillance study. Antimicrob Agents Chemother 1999; 43: 1901-8.

3.- National Committee for Clinical Laboratory Standards. Performance standards for antimicrobial susceptibility testing. Twelve informational supplement M100-S12. Wayne, Pennsylvania: NCCLS, 2002.

4.- PALAVECINO E L, RIEDEL I, BERRIOS X et al. Prevalence and mechanisms of macrolide resistance in Streptococcus pyogenes in Santiago, Chile. Antimicrob Agents Chemother 2001; 45: 339-41.

5.- FASOLA E L. BAJAKSOUZIAN S, APPELBAUM $\mathrm{P}$ C, JACOBS M R. Variation in erythromycin and clindamycin susceptibility testing of Streptococcus pneumoniae with four test methods. Antimicrob Agent Chemother 1997; 41: 129-34.

6.- NAGAI K, DAVIES T, EDNIE L et al. Activities of a new fluoroketolide HMR 3787 and its (des)-fluor-derivative RU 64339 compared to telithromycin, erythromycin, azithromycin, clarithro-mycin and clindamycin against a variety of macrolide susceptible and resistant Streptococcus pyogenes. Antimicrob Agents Chemother 2001; 45: 3242-5.

Correspondencia a:

Elizabeth Palavecino Rosales

Email: epalavecino@hotmail.com 